than resveratrol in inhibiting chemotaxis of HL-60 cells and blocking cell cycle of THP-1 and HL-60 cells at G1/S transition. In addition, NBT-167, but not resveratrol, could increase IL-2 production and $\mathrm{T}$ cell proliferation stimulated with antiCD3 and anti-CD28 and synergize with anti-PD-1 antibody to increase IL-2 and IFN-gamma production in co-culture of allotypic T cells and dendric cells (MLR).

Conclusions Our data showed that NBT-167, a dimer of resveratrol, had anticancer and immunomodulatory activities such as modulation of expression of cytokines in immune cells and induction of cancer cell-killing activities of NK and gamma delta T cells. Generally, NBT-167 appeared to have higher activities than resveratrol in modulating immune cells and inhibiting cancer cells. NBT-167 could be a promising cancer immunotherapeutic agent targeting both cancer cells and immune cells.

http://dx.doi.org/10.1136/jitc-2020-SITC2020.0600

\section{DEVELOPMENT OF IMPROVED SMALL MOLECULE STING AGONISTS SUITABLE FOR SYSTEMIC ADMINISTRATION}

Maciej Rogacki*, Stefan Chmielewski, Magdalena Zawadzka, Jolanta Mazurek, Katarzyna Wnuk-Lipińska, Kamil Kuś, Karolina Gluza, Katarzyna Wójcik-Jaszczyńska, Aleksandra Poczkaj, Lukasz Dudek, Wojciech Schonemann, Urszula Głowniak-Kwitek, Marcin Leś, Marek Wronowski, Tushar Mahajan, Urszula Kulesza, Magdalena Zastawna, David Synak, Karol Zuchowicz, Katarzyna Banaszak, Karolina Wiatrowska, Izabela Strojny, Mirosława Gładysz, Justyna Jabłońska, Ewelina Gabor-Worwa, Raghuram Tangirala, Luigi Stasi, Peter Littlewood, Krzysztof Brzózka, Monika Dobrzańska. Ryvu Therapeutics, Kraków, Poland

Background Stimulator of Interferon Genes (STING) is a major player in the activation of robust innate immune response leading to initiation and enhancement of tumor-specific adaptive immunity. Several clinical and pre-clinical programs have shown that activation of the STING pathway triggers immune-mediated antitumor response. Although vast majority of programs focus on development of analogues of the endogenous STING ligands, their chemical nature and stability often limit their use to local administration. Herein, we present recent results from the development of our selective non-nucleotide, non-macrocyclic, small molecule direct STING agonists, suitable for systemic administration, characterized by improved activity in human immune cells.

Methods Binding to recombinant STING protein was examined using FTS, MST, FP and crystallography studies. Phenotypic screen was performed in THP-1 Dual reporter cells. Mouse bone marrow-derived dendritic cells (BMDC) were obtained from C57BL/6 mice and differentiated with mIL-4 and mGM-CSF. STING agonists were administered into BALB/ $c$ mice and cytokine release was measured in plasma. Additionally, mice were inoculated with CT26 murine colon carcinoma or EMT6 murine breast carcinoma cells and the compound was administered, followed by the regular tumor growth and body weight monitoring.

Results Ryvu's small-molecule agonists demonstrate strong binding affinity to recombinant STING proteins across all tested species. The compounds bind to all human STING protein variants and trigger pro-inflammatory cytokine release from human immune cells regardless of the STING haplotype. Moreover, new generation of developed agonists show significantly improved binding to human protein as well as in vitro activity on human cells. Systemic, intravenous in vivo administration leads to a dose-dependent upregulation of STING- dependent pro-inflammatory cytokines, which results in a dose-dependent antitumor efficacy observed in CT26 and EMT6 mouse cancer models, leading to complete tumor remissions in all treated animals. Furthermore, observed efficacy is accompanied by development of a lasting immunological response demonstrated by lack of tumor engraftment or a delayed tumor growth in cured animals challenged with repeated inoculation of cancer cells.

Conclusions New generation Ryvu's STING agonists are strong and selective activators of STING-dependent signaling in both mouse and human immune cells promoting anti-tumor immunity. Treatment with Ryvu's small-molecule STING agonists leads to engagement of the immune system which results in a complete tumor remission and development of immunological memory of the cancer antigens. The compounds show good selectivity and ADME properties enabling development for systemic administration. In addition developed compounds maintain small functional handles amenable to linker attachment making the series suitable for versatile development as single agents, for combinations with immunotherapies or as targeted agents.

http://dx.doi.org/10.1136/jitc-2020-SITC2020.0601

\section{STING AGONIST-BASED TREATMENT PROMOTES VASCULAR NORMALIZATION AND TERTIARY LYMPHOID STRUCTURE FORMATION IN THE THERAPEUTIC MELANOMA MICROENVIRONMENT}

Manoj Chelvanambi*, Ronald Fecek, Jennifer Taylor, Walter Storkus. University of Pittsburgh, Pittsburgh, PA, USA

Background The degree of immune infiltration in tumors, especially CD8 $+\mathrm{T}$ cells, greatly impacts patient disease course and response to interventional immunotherapy. Hence, enhancement of TIL prevalence is a preferred clinical endpoint, one that may be achieved via administration of agents that normalize the tumor vasculature (VN) leading to improved immune cell recruitment and/or that induce the development of local tertiary lymphoid structures (TLS) within the tumor microenvironment (TME).

Methods Low-dose STING agonist ADU S-100 (5 $\mu \mathrm{g} /$ mouse) was delivered intratumorally to established s.c. B16.F10 melanomas on days 10, 14 and 17 post-tumor inoculation under an IACUC-approved protocol. Treated and control, untreated tumors were isolated at various time points to assess transcriptional changes associated with VN and TLS formation via qPCR, with corollary immune cell composition changes determined using flow cytometry and immunofluorescence microscopy. In vitro assays were performed on CD11c+ BMDCs treated with $2.5 \mu \mathrm{g} / \mathrm{mL}$ ADU S-100 (vs PBS control) and associated transcriptional changes analyzed via qPCR or profiled using DNA microarrays. For TCR $\beta$-CDR3 analyses, CDR3 was sequenced from gDNA isolated from enzymatically digested tumors and splenocytes.

Results We report that activation of STING within the TME leads to slowed melanoma growth in association with increased production of angiostatic factors including Tnfsf15 (Vegi), Cxcl10 and Angpt1, and TLS inducing factors including Ccl19, Ccl21, Lta, Ltb and Tnfsf14 (Light). Therapeutic responses from intratumoral STING activation were characterized by increased vascular normalization (VN), enhanced tumor infiltration by CD8 $+\mathrm{T}$ cells and CD11c+ DCs and local TLS neo-genesis, all of which were dependent on host 